\title{
El proceso de industrialización en Baja California
}

\author{
Agustín Sández Pérez \\ Instituto de Investigaciones Sociales \\ Universidad Autónoma de Baja California
}

\begin{abstract}
Resumen
El presente trabajo plantea como propósito central indagar sobre uno de los periodos del desarrollo industrial bajacaliforniano. Este ensayo aborda el papel de la política gubernamental y su efecto sobre la estructura económica de esta región fronteriza. Su historia económica presenta diversas modalidades de participación del capital extranjero. Los enfoques y actitudes del gobierno mexicano en su relación con los Estados Unidos han resultado cada vez más determinantes sobre los acontecimientos económicos regionales.
\end{abstract}

Palabras clave: proceso de industrialización, política gubernamental, capital extranjero, economía regional, zona libre.

\begin{abstract}
This research proposes to discover the facts related to the periods of time of the industrial development of Baja California. It also offers arguments about the role of the government politics regarding its effect on the economical structure of this border region. The economical history presents diverse forms of share on foreign capitals. The diverse methods used by Mexican government to focus and work on the relations with the United States have conditioned the regional economical events.
\end{abstract}

Keywords: industrialization process, governmental politics, foreign capital, regional economics, free zone. 


\title{
EL PROCESO DE INDUSTRIALIZACION EN BAJA CALIFORNIA ${ }^{1}$
}

\author{
Por: \\ Agustín Sández Pérez
}

La presente exposición tiene como propósito el desarrollo de un marco general explicativo de los principales cambios ocurridos en la actividad industrial bajacaliforniana en tres amplios períodos históricos. El primero, comprendido entre fines del siglo pasado y mediados de la tercera década del presente, en que las políticas del gobierno mexicano impulsaron la colonización de la península sobre la base de la inmigración extranjera; el segundo, que va de la promulgación del decreto de Zona Libre hasta mediados de los sesentas, en que se formalizan las condiciones de libre interacción de la zona con los Estados Unidos y; un tercero, a partir del año de 1965, en que se establece el así llamado Programa de Industrialización Fronteriza, a partir del cual, y no sin altibajos, la actividad maquiladora se convierte en el eje de la estrategia económica fronteriza.

La política económica ha jugado un papel central en la determinación de las tendencias observadas en cada etapa. Sus efectos han sido notables sobre la vida económica en la frontera,pero muy especialmente sobre el acontecer de la actividad industrial. Si bien es cierto que la ubicación geográfica confiere a la región una naturaleza particular, a lo largo de la exposición se desarrolla el argumento de que en realidad las estrategias económicas han sido capaces de desatar procesos, que siendo potenciales, alcanzan una expresión concreta sólo bajo condiciones específicas de regulación. Ello explica cómo, a lo largo de distintas fases, las tendencias no hayan sido las mismas ni mucho menos la intensidad con que operaron. En especial, se propone aquí que las medidas gubernamentales a nivel central han magnificado sus efectos en épocas más recientes.

* Investigador del Instituto de Investigaciones Sociales de la Universidad Autónoma de Baja California.

1 El presente ensayo desarrolla y complementa algunos argumentos presentados en el capítulo primerodel documento "Estructura y dinámica del sector manufacturero bajacaliforniano 1960-1985". Cuadernos de Ciencias Sociales. Serie 3 No. 7 IIS-UABC. Mexicali 1987. 


\section{Período (1880-1937) Surgimiento de formas iniciales de acumulación en el sector industrial.}

Desde tiempos remotos, los asentamientos poblacionales en Baja California se localizaron en el sur de la península. Sólo a fines del siglo pasado la región septentrional conoce los primeros esfuerzos por establecer algunos centros de población, asociado esto con los movimientos migratorios que desde la costa este de los Estados Unidos, así como de Europa y Asia, se desplazan al oeste norteamericano. $\mathrm{El}$ incipiente crecimiento de la costa occidental se convirtió en el principal atractivo para la colonización de Californiay en consecuencia del norte de la península. ${ }^{2}$

Ensenada surge como la primera localidad de importancia económica en el entonces Distrito Norte de la Baja California. Según datos del censo de población de 1888, era considerable la presencia de personas de otras nacionalidades, especialmente americanos, chinos, italianos, alemanes; muchos de ellos dedicados a actividades agrícolas, mineras o comerciales. Es significativo que su presencia fuese casi absoluta en la naciente y escasa actividad industrial que surgía entonces. ${ }^{3}$ La ley de colonización de 1884 había exceptuado del pago de impuestos a los colonos; sin embargo, agentes fiscales llegaron a exigir en ocasiones el pago de derechos, con lo que algunas personas prefirieron establecerse en San Diego y en otras partes de los Estados Unidos. La aduana marítima de Ensenada comenzó a pagar con ingresos propios a sus empleados, lo que sucedió igualmente en San Quintín, Punta Banda y otros lugares. (Walther Meade, 1986: 87).

Las primeras décadas de este siglo presenciaron el surgimiento de nuevas formas de actividad económica en Ensenada y Tijuana. Se asiste al desarrollo de los primeros esfuerzos en la transformación de bienes de origen regional así como de importación. La característica

2 “...no había parte de la frontera de nuestro país que invitara la inmigración norteamericana tanto como el Distrito Norte..Enumerando las causas que influyeron en el crecimiento poblacional del vecino país expuso (Luis E. Torres, jefe político) que la población del norte y el este emigraba en el invierno a las costas de California en busca de un clima benigno.Que esa población, al principio flotante, se había radicado posteriormente y extendido por toda la costa..."(Walther Meade, 1986: 94).

3 "...algunos extranjeros habían establecido en Ensenada algunas industrias como una fábrica de jabón, una de aguas gaseosas, una de cerveza, una de puertas y ventanas, una de envase de frutas en su jugo y otra más... Carlos Benett, establecido en el valle de Maneadero, manejaba una fábrica de conservas, un aserradero y el abasto de agua de la ciudad.." (Walther Meade. 1986: 92 y 83). 
dominante en la etapa previa había sido justamente la escasa generación de valor agregado debido a las estrechas ataduras que la producción mantenía con el sector primario y extractivo. Ensenada contaba con cierto grado de diversificación industrial; sin embargo, apenas se lograba satisfacer una escasa proporción de las necesidades básicas de la población. La situación era parecida en el resto de las localidades. ${ }^{4}$ En forma paralela, sobre todo en el caso de Tijuana, se gesta un importante movimiento comercial vinculado con los desplazamientos turísticos provenientes de California $y$, crecientemente, de otros lugares de vecino país.

El valle de Mexicali no registró actividad industrial de significación sino hasta mediados de los años veintes. Su producto agrícola en los primeros lustros del siglo fue apenas importante. Ello se debió a que se trataba de tierras en propiedad de extranjeros quienes se interesaban más con fines especulativos que por su potencialidad de producción. ${ }^{5}$ Si bien es cierto que se cultivó algodón en esos años, la etapa de mayor desarrollo correspondió a una muy posterior, en que la participación del capital extranjero se llevó a cabo en forma indirecta mediante el control de la comercialización de la producción agrícola, una vezrealizada la expropiación por el presidente Cárdenas. ${ }^{6}$ Durante los años veinte, fue notable la presencia de la industria cervecera en Mexicali, así como la producción de vinos y licores en Tijuana y Ensenada. Esto representó la presión principal para el establecimiento de la primera restricción a los perímetros libres en 1934. ${ }^{7}$

4 “...en 1917, (al declarar Estados Unidos el estado de guerra en la Primera Guerra Mundial, esto se reflejo directamente) en las poblaciones mexicanas de la frontera, especialmente en Mexicali, que ya desde entonces dependía totalmente de los productos alimentarios americanos" (Aguirre Bernal, s/f: 172).

5 “...el crecimiento del valle de Mexicali estuvo ligado al ritmo lento que le marcó la California-México Land \& Cattle Company. Por dos razones: primero, la compañía abrí́ pocas tierras al cultivo; segundo, se negaba a vender dichos terrenos" (Zazueta, 1978: 49).

6 "Con la nacionalización de la tierra -y posteriormente del agua- se da una relativa recuperación de la producción agrícola, pero el capital extranjero pierde la propiedad de la tierra y el control directo del proceso de producción... aunque no tiene presencia masiva en la zona y sólo tiene intereses en algunas compañías algodoneras su presencia resulta significativa considerando que la actividad económica derivada del cultivo del algodón es, con mucho, la más importante" (Stamatis, 1987).

7 "El 30 de mayode 1934 se estableció la primera restricción fijándose un impuesto especial a todas la bebidas que se introdujeran, para proteger la producción vinícola y cervecera regional" (Centro de Investigaciones Históricas UNAM-UABC,1983: 540). 
La situación de la actividad industrial, conforme a los datos censales obtenidos en 1930, correspondía a un estado de desarrollo apenas significativo, en el que predominaba la producción alimentaria, destacando pescadoy mariscos junto con la producción cervecera.

Durante esta etapa se asiste a un cambio importante en la configuración regional, a partir del decaimiento de la vida industrial ensenadense y del mayor despegue comercial de las poblaciones contiguas a la línea fronteriza. En este proceso, el cambio de actitud de la política oficial, al promover el libre juego de las fuerzas de mercado, parece haber desempeñado un rol crucial.

La situación de las industrias nacientes era sensiblemente afectada por la libre importación, obligándolas a solicitar protección a fin de poder desarrollar economías internas. Las actividades agroindustriales en Mexicali giraron alrededor del sistema algodonero, cobrando gran importancia en los treintas (Centro de Investigaciones Históricas, 1983: 159-160) junto con la elaboración de aceites y grasas vegetales, enlatado de pescado y mariscos, elaboración de vino y licores, embotellado de aguas gaseosas, empaque de carne y pasteurización de leche representaron las principales actividades ya existentes antes del Decreto de Zona Libre.

\section{Período (1937-1965): La Zona Libre y la nueva estrategia política de colonización nacional.}

Con el Decreto de Zona Libre en 1937 se formaliza el régimen fiscal de excepción para la zona. La medida se ha interpretado en alguna ocasión como una compensación por el hecho de haber expropiado las tierras del valle de Mexicali a los propietarios extranjeros. ${ }^{8}$

De hecho, representó un elemento crucial en la nueva estrategia de colonización, permitiéndole la creación de un subsistema económico periférico del sistema central que mantendría, por un plazo largo, condiciones preferenciales en materia de ingresos personales -sobresueldos, primas, etc.-, además del "atractivo" del consumo generalizado de todo tipo de bienes de procedencia norteamericana. Debe considerarse que el problema principal que

8 “..en un movimiento que al parecer fue de compensación política, Cárdenas decretó el 25 de junio de 1937 el régimen de Zona Libre en el entonces Territorio Norte de Baja California, al tiempo que afectaba los terrenos de la Colorado River Land Company" (Tamayo y Fernández, 1983: 70). 
enfrenta entonces la política del gobierno de la República, era la insuficiente incorporación de nacionales entre los grupos interesados en colonizar esta región. Sólo una estrategia deliberada, en que probablemente tuvieran que sacrificarse objetivos económicos en aras de una recuperación del territorio, haría posible el desencadenamiento de tal proceso. En este sentido, la implantación de la Zona Libre se convierte en el eje de una estrategia política de soberanía nacional.

En esta etapa se inicia la sustitución de importaciones en algunos bienes de consumo durable e intermedio. La producción agrícola alcanzaba su mayor auge, sobre todo en la década de los cincuentas. El crecimiento del comercio fue impresionante. Todo ello significó un enorme impulso a la colonización regional, sostenida en un crecimiento económico dependiente del exterior, ya fuera por el mercado de exportación del algodón o bien por la demanda asociada con la actividad turística en el comercio y los sevicios.

El sector agrícola fue objeto de una creciente capitalización -permitiendo elevar los rendimientos- que, conjuntamente con las condiciones favorables del mercado de exportación, explica el surgimiento de un excedente económico sin precedente que pasa a incorporarse en un esquema de acumulación que, partiendo de una base agrícola -rural-, empieza a gravitar crecientemente sobre el espacio urbano. Fue éste un período en que se amasaron grandes fortunas. ${ }^{9}$ La Zona Libre creó un medio propicio para la capitalización de amplios sectores productivos y sin embargo, al mismo tiempo, condicionó las posibilidades de realización del potencial correspondiente a la capacidad instalada en la economía. Lo anterior se sumó a las limitaciones aduaneras para la introducción de productos regionales al centro del país, levantándose así la principal "barrera a la entrada" a empresas de otras regiones y a la "expansión", en el caso de firmas regionales. De este modo, el sector industrial, a pesar de su alto grado de especialización, en muchos casos no logróbeneficiarse de economías dinámicas a escala.

El régimen propició una expansión y diversificación casi indiscriminada en el caso de las actividades comerciales. Se importaron alimentos, artículos para el hogar, combustibles, vestidos, transportes,

9 "En 1950, al invertirse las condiciones en Estados Unidos, la industrialización de sus vecinos californianos se ve impulsada por el boom de la posguerra. La principal actividad productiva de la zona, el cultivo del algodón, alcanza una prosperidad nunca vista con anterioridad, debidoa la recuperación del mercado internacional" (Zazueta, 1978: 128). 
bebidas, materiales de construcción, entre otros. Las actividades financieras entablaron importantes líneas crediticias entre ambos lados de la frontera.

En esta etapa la economía de la región observa un alto índice de crecimiento, como resultado del desempeño de su base exportadora. Esta situación no fue en modo alguno particular a la zona, pues lo mismo ocurría con la actividad agrícola comercial de orientación externa en el resto del país.

Existía un desfase en relación al avance que pudoalcanzarse en otras regiones en materia de sustitución de importaciones; sin embargo, el contexto económico compartía con el interior el hecho de crecer vía la profundización de la dependencia externa.

A lo largo de este período se observa un cambio importante del eje geográfico del proceso de acumulación en sentido horizontal, de occidente a oriente, en virtud de la importancia de la exportación algodonera así como de las actividades que participaron dentro del complejo textil. Esto significó la aparición de la economía industrial mexicalense en el panorama regional. ${ }^{10}$ Las determinaciones geográficas empezaron a ceder.

Período (1965-1987): La maquila. Una estrategia económica de generación de empleos en la frontera.

Hacia mediados de los sesentas era ya visible el agotamiento del modelo primario monoexportador. Las condiciones de mercado del algodón en el exterior resultaban sumamente desfavorables, entre otras cosas, debido al surgimiento de productos sustitutos cercanos, fibras sintéticas. Por otro lado, la suspensión del programa bracero alarmó a ciertos sectores de opinión en México acerca de las posibles consecuencias sociales que esta medida tendría en la frontera. La política oficial, atendiendo estas cuestiones y habiendo revisado algunas de las experiencias que surgieron en otros países, implementó el llamado Programa de Industrialización Fronteriza -después del fracaso del Programa Nacional Fronterizo-, el cual tuvo resultados a muy corto plazo al permitir la implantación de un número considerable

10 "Desde 1945 en adelante (Mexicali) experimenta un saludable proceso de industrialización basadoen la transformación de recursos naturales y materias primas... el municipio concentrará para 1955 el $80 \%$ de la producción industrial del estado y reunirá el 75\% de la inversión industrial de Baja California" (Zazueta, 1982: 100). 
de plantas ensambladoras, la mayoría de capital norteamericano, a lo largo de las principales ciudades en la franja contigua a los Estados Unidos.

CUADRO 1. Estructura industrial delas ciudades en B.C. (1960-1975)

Composición industrial (1960) según valor agregado Rama/ciudad Mexicali Tijuana Tecate Ensenada B.C.

\begin{tabular}{lrrlrr}
\hline Alimentos & 44.4 & 46.7 & n.d. & 64.1 & 50.1 \\
Bebidas & 24.3 & 18.6 & n.d. & 7.4 & 18.6 \\
Textiles & 6.8 & - & n.d. & - & 3.6 \\
Vestido & 0.7 & 5.0 & n.d. & 1.1 & 1.7 \\
P metálicos & 0.3 & 0.9 & n.d. & 2.2 & 0.9 \\
M y e electr & 2.5 & 2.3 & n.d. & 0.3 & 1.9 \\
E transporte & 4.6 & 3.9 & n.d. & 0.3 & 3.4 \\
Otros & 16.4 & 22.6 & n.d. & 24.6 & 19.8 \\
\hline
\end{tabular}

Composición industrial (1965)

según valor agregado

Rama/ciudad Mexicali Tijuana Tecate Ensenada B.C.

\begin{tabular}{lrrrrr}
\hline Alimentos & 43.5 & 20.8 & 8.3 & 63.5 & 41.3 \\
Bebidas & 11.8 & 15.1 & 89.7 & 8.2 & 14.8 \\
Textiles & 15.5 & 24.9 & - & - & 9.0 \\
Vestido & 1.1 & 7.2 & n.s. & 0.9 & 2.4 \\
P metálicos & - & 5.5 & - & 10.2 & 3.6 \\
M y e electr & 2.2 & 1.6 & 0.7 & 0.4 & 1.2 \\
E transporte & 9.6 & 0.1 & - & 2.0 & 6.9 \\
Otros & 16.3 & 24.8 & 1.3 & 14.8 & 20.8 \\
\hline
\end{tabular}

Composición industrial (1970)

según valor agregado

Rama/ciudad Mexicali Tijuana Tecate Ensenada B.C.

\begin{tabular}{lrrrrr}
\hline Alimentos & 24.4 & 17.6 & 2.8 & 49.5 & 22.9 \\
Bebidas & 5.7 & 6.4 & 87.5 & 6.1 & 19.1 \\
Textiles & 7.0 & 7.9 & - & - & 5.5 \\
Vestido & 5.6 & 5.0 & n.s. & 1.1 & 4.0 \\
P metálicos & 1.5 & 6.4 & 2.4 & 16.5 & 5.4 \\
M y e electr & 10.6 & 23.0 & 5.0 & 0.3 & 12.9 \\
E transporte & 18.5 & 0.2 & - & - & 7.4 \\
Otros & 26.7 & 33.5 & 2.3 & 26.5 & 22.8
\end{tabular}




\section{CUADRO 1. (Continuación)}

Composición industrial (1975)

según valor agregado Rama/ciudad Mexicali Tijuana Tecate Ensenada B.C.

\begin{tabular}{lrrrrr}
\hline Alimentos & 19.9 & 20.5 & 2.0 & 59.1 & 25.0 \\
Bebidas & 2.8 & 6.2 & 91.2 & 7.1 & 19.1 \\
Textiles & 3.8 & 1.2 & - & - & 1.7 \\
Vestido & 4.2 & 6.5 & 0.5 & 0.3 & 3.4 \\
P metálicos & 3.9 & 6.7 & 0.2 & 17.0 & 6.7 \\
M y e electr & 13.5 & 25.8 & 3.4 & 1.8 & 12.6 \\
E transporte & 32.3 & - & - & - & 11.9 \\
Otros & 19.6 & 33.1 & 2.7 & 14.7 & 19.6 \\
\hline
\end{tabular}

El crecimiento vía maquiladora significó una reorientación del patrón de industrialización hacia sectores cuya vinculación con el resto de las actividades manufactureras es prácticamente nula. En contraste, la experiencia histórica de industrialización de muchos países desarrollados significó un proceso de generación de complejos económicos eslabonados interindustrialmente-, que hicieron asequibles externalidades y economías de aglomeración. Si bien sus modalidades implicaron con frecuencia patrones polarizados, éstos fueron geográficamente dispersos.

El crecimiento de la actividad industrial en la frontera mexicana con los Estados Unidos ha correspondido a una modalidad de enclave que minimiza la capacidad de dinamización de otros sectores, en tanto no se acompaña de una estrategia complementaria que permita generar insumos y además abatir las necesidades básicas del creciente contingente de fuerza de trabajo.

Dado el escaso grado de diversificación observado antes de los años sesentas, la apertura del programa maquilador implicó en el corto plazo un efecto significativo sobre la estructura industrial de la entidad. Empero, pocos años bastaron para hacer visible la dependencia funcional del nivel de actividad de dicho sector con el nivel de salario real existente en el país medido en dólares. Ello explica la contención en las tendencias de diversificación hacia mediados de los sesentas.

A partir de 1976, pero especialmente después de 1982, puede observarse claramente cómo la intensificación del proceso maquilador se encuentra atado al creciente deterioro de la retribución salarial y al margen de subvaluación de la paridad peso-dólar. 
CUADRO 2. Crecimientode la industria maquiladora de exportación en Baja California

1974-1986 Baja California

\begin{tabular}{lrrrrrrrr} 
Ciudad & \multicolumn{2}{c}{ Tijuana } & \multicolumn{2}{c}{ Mexicali } & \multicolumn{2}{c}{ Tecate } & \multicolumn{2}{c}{ Ensenada } \\
Año & N.E & P.O & \multicolumn{2}{c}{ N.E } & P.O & N.E & P.O & \multicolumn{2}{c}{ N.E } & P.O \\
\hline 1974 & 101 & 9276 & 57 & 7888 & 11 & 1098 & 5 & 220 \\
1975 & 99 & 7844 & 67 & 6324 & 10 & 803 & 6 & 314 \\
1976 & 93 & 7795 & 69 & 6604 & 13 & 717 & 6 & 163 \\
1977 & 92 & 7111 & 70 & 6351 & 11 & 774 & 5 & 160 \\
1978 & 95 & 8778 & 65 & 6543 & 15 & 640 & 4 & 154 \\
1979 & 101 & 10889 & 77 & 7965 & 20 & 560 & 5 & 275 \\
1980 & 123 & 12343 & 79 & 7146 & 22 & 572 & 6 & 257 \\
1981 & 127 & 14482 & 64 & 7628 & 20 & 805 & 4 & 267 \\
1982 & 124 & 14959 & 54 & 6268 & 17 & 783 & 5 & 223 \\
1983 & 131 & 17423 & 55 & 7392 & 19 & 1168 & 6 & 268 \\
1984 & 147 & 23047 & 67 & 10264 & 25 & 1606 & 9 & 340 \\
1985 & 192 & 25913 & 75 & 10876 & 31 & 1755 & 9 & 431 \\
1986 & 238 & 30248 & 86 & 12727 & 33 & 2366 & 9 & 547 \\
\end{tabular}

Nota: N.E número de establecimientos / P.O personal ocupado

Fuente: SPP-INEGI, Estadística de la industria maquiladora de exportación, 1987.

Las pequeñas y medianas empresas agroindustriales han mantenido lazos con la producción agrícola regional; sin embargo, enfrentan en forma persistente la competencia con productos de origen norteamericano.

El margen de subvaluación, junto con la presencia de nuevos canales de comercialización, ha permitido una alteración sensible -si bien coyuntural- en los patrones de compra de un sector considerable de la población, a la vez que ha estimulado la producción y abasto de bienes de consumo no-durables. La situación difiere notablemente en el caso de bienes de difícil sustitución -intermedios y de capital que representan necesidades de importación, castigadas por la subvaluación,lesionándose la adecuación tecnológica de varias actividades de transformación. La estrategia oficial de liberación comercialcon el exterior ha minimizado las diferencias prevalecientes en el funcionamiento económico de la zona respecto al del interior del país. 


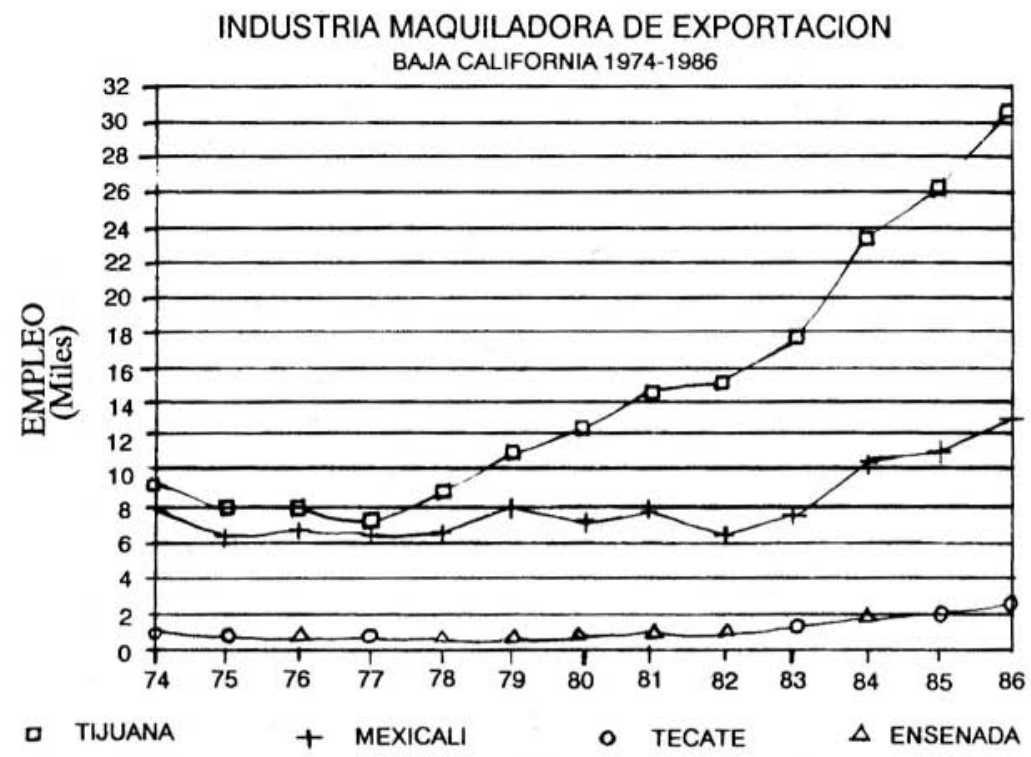

FIGURA 1. Industria maquiladora de exportación Baja California (1974-1986)

\section{CONCLUSION}

La historia de la industrialización bajacaliforniana presencia diversas modalidades de participación del capital extranjero. La península, antes de representar un objeto económico, significó un espacio político en el que se arbitraron las estrategias nacionales de colonización frente a los intereses de expansión territorial norteamericanos a los largo de casi un siglo, transcurrido entre los Tratados de Guadalupe-Hidalgo y la política cardenista de recuperación nacional.

Durante el porfiriato se promovióla presencia de capital extranjero argumentando, entre otras cosas, el problema de la deuda externa y las necesidades de desarrollo regional. En esa etapa, la incipiente actividad industrial sólo existió en Ensenada, generalmente vinculada con la producción agrícola y minera. El surgimiento del proceso revolucionario en el interior del país promovió una nueva actitud frente al capital extranjero, lo cual alcanzó su máxima expresión con las expropiaciones del presidente Cárdenas en los treintas. 
En Baja California el surgimiento de la Zona Libre se convirtió en el eje de una estrategia de colonización nacional,que pudo concretarse sólo a cambio de concesiones importantes en materia de integración económica. Se presentó así una etapa de alto crecimiento económico basado en la profundización de la dependencia externa, la cual registró especificidades locales pero de ningún modo fue particular a la región.

Una vez garantizado el poblamiento de la zona y dada la existencia de una importante corriente migratoria hacia el norte del país, el Programa de Maquiladoras se convierte en el eje de los procesos productivos de localización fronteriza.

Los acontecimientos regionales, dentro de su especificidad histórica, se encuentran fuertemente correlacionados con los enfoques y actitudes del estado mexicano en su relación con los Estados Unidos. La determinación de la estrategia político-económica sobre la situación fronteriza ha sido cada vez más evidente. 


\section{B I B L I O G R A F I A}

AGUIRRE, Bernal Celso. s/n, Compendio histórico bigráfico de Mexicali 1539-1966. Mexicali. 3ra. ed.

CENTRO DE INVESTIGACIONESHISTORICAS, 1983.Panorama histórico de Baja Califomia. UNAM-UABC.

STAMATIS Maldonado, Martha. 1987 "El valle de Mexicali: agricultura e inversión extranjera". Ponencia presentada en el I EncuentroSobre Problemas del Campo en el Noroeste de México y el Suroeste de los Estados Unidos. Culiacán,Sin. México. Enero de 1987.

TAMAYO, Jesús y Fernández, José Luis. 1983 Zonas fronterizas. CIDE.

WALTHER MEADE, Adalberto. 1986 El Partido Norte de la Baja California. UABC. Mexicali, B. C.

ZAZUETA, Carlos. 1978 La formación de la Frontera Norte. Tesis de Maestría. Centro de Estudios Industriales. El Colegio de México. México.

ZAZUETA, Carlos. 1982 Mexicali: su historia, la ciudad, sus actividades y sus hombres. CENIET-STPS. México. 